\title{
Squeezed cooling of mechanical motion beyond the resolved-sideband limit
}

\author{
Lin Zhang and Cheng Yang \\ School of Physics and Information Technology, Shaanxi Normal University, Xi'an 710061, P. R. China \\ Weiping Zhang
Department of Physics and Astronomy, Shanghai Jiao Tong University, Shanghai 200240, P. R. China
}

\begin{abstract}
Cavity optomechanics provides a unique platform for controlling micromechanical systems by means of optical fields that crosses the classical-quantum boundary to achieve solid foundations for quantum technologies. Currently, optomechanical resonators have become promising candidates for the development of precisely controlled nano-motors, ultrasensitive sensors and robust quantum information processors. For all these applications, a crucial requirement is to cool the mechanical resonators down to their quantum ground states. In this paper, we present a novel cooling scheme to further cool a micromechanical resonator via the noise squeezing effect. One quadrature in such a resonator can be squeezed to induce enhanced fluctuation in the other, "heated" quadrature, which can then be used to cool the mechanical motion via conventional optomechanical coupling. Our theoretical analysis and numerical calculations demonstrate that this squeeze-and-cool mechanism offers a quick technique for deeply cooling a macroscopic mechanical resonator to an unprecedented temperature region below the zero-point fluctuations.
\end{abstract}

PACS numbers:

Cavity optomechanics [1] concerns the strong interactions between optical fields and mechanical oscillators that are derived from the mechanical effects of photons. Radiation pressure is a major light-induced mechanical force arising from the fact that a massless photon carries momentum. Linear or angular momentum can be transferred from light fields to mechanical objects when photons are absorbed or emitted. However, the mechanical effect of radiation pressure on a macroscopic object is extremely weak [2]. Fortunately, high- $Q$ optical cavities can resonantly enhance this optical force by trapping high-intensity light within a very small volume, such as a typical linear Fabry-Pérot cavity consisting of a heavy fixed mirror and a light movable mirror attached to an elastic boundary (see Fig 1] [1. Since a photon is reflected multiple times (e.g., $10^{6}[3]$ ) between the two cavity mirrors before it decays, an intense cavity field builds up, resulting in a large optical force on the movable mirror, which causes the mirror to vibrate at a frequency ranging from $\mathrm{kHz}$ to $\mathrm{GHz}$ [4]. In recent decades, the field of cavity optomechanics has witnessed rapid growth, and related research has become increasingly important for both fundamental physics and applied technology [5].

Efficient cooling of a massive mechanical resonator to its quantum ground state is a prominent achievement of cavity optomechanics 6 8 , and a mechanical resonator with an average phonon occupation of $0.20 \pm 0.02$ has recently been achieved [9]. Cooling an oscillation mode to the ground state with a noise below the standard quantum limit is a fundamental requirement for various optomechanical applications, such as reliable nano-motors, high-precision sensors and robust quantum processors. The basic physics of conventional optomechanical cooling is that the coupled cavity field introduces additional friction to adaptively reduce the momentum of the resonator via an optical-spring effect [10, 11]. However, the quan- tum uncertainty principle prevents the complete halting of the resonator's motion to access a temperature beyond the quantum fluctuations. In the attempt to achieve deeper cooling towards quantum limit [12, the typical mechanism based on dynamical backaction loses efficacy due to the quantum backaction limit, and various alternative cooling schemes within the resolved-sideband limit have been proposed to achieve lower temperatures [1316. Although a recent cooling experiment using squeezed light have overcome the quantum backaction limit [17, all sideband cooling techniques are eventually limited by the single-phonon scattering balance 9], which makes ground-state cooling below the one-phonon level more difficult due to a low final efficiency.

In this paper, we present an alternative technique for deep cooling via squeezing. The basic idea of this technique is very similar to that of magnetic refrigeration. The confinement of magnetic dipoles in one direction by an external magnetic field will drive fluctuations in the spatial degrees of freedom into the momentum channel, thereby effectively improving the temperature of the refrigerant. This thermal squeezing effect can be essentially understood in terms of an oscillator entering a tighter potential with a more confined position, causing its momentum to increase. Thus, the problem becomes one of cooling a hotter refrigerant, which is easier than cooling a cold one. When the confinement is finally removed, the motion fluctuations will return back to the spatial degrees of freedom, and the overall temperature will decrease. Similarly, in the quantum domain, when one quadrature of a mechanical resonator is squeezed, the other conjugate quadrature will be "heated" by increased quantum fluctuations due to the quantum uncertainty principle [18. For a given optomechanical oscillator, the quantum fluctuations in the momentum quadrature $p$ can be increased by quantum squeezing on the position quadra- 
ture $x$. Then, the "hotter" quadrature, with its amplified fluctuations, can be directly coupled to a blue-detuned cavity mode for conventional optomechanical cooling.

The quantum noise squeezing effect and its applications have been understood for approximately 50 years [19, 20], and noise squeezing on optomechanical resonators has also been achieved using various schemes 21 25. Because parametric squeezing is a robust technique applied in many quantum and classical systems [26 29 , we choose parametric driving as our means of creating mechanical squeezing and theoretically study the cooling scheme for an optomechanical oscillator that is squeezed by parametric amplification [30. Our theoretical analysis shows that this squeezed resonator can be effectively cooled down to a low temperature that is limited only by the degree of squeezing. With repeated cycles of squeezing and cooling, the resonator can be deeply cooled to a temperature region that is inaccessible for conventional cooling methods without noise squeezing.

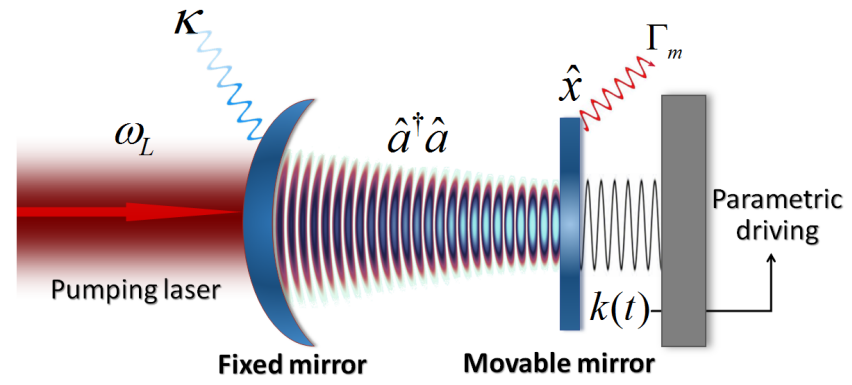

FIG. 1: Schematic diagram of a hybrid optomechanical system with a parametric mirror independently driven by the modulation of its spring constant $k(t)$ and coupled to a cooling cavity mode.

The main schematic of our system, which consists of a high- $Q$ cavity and a movable mirror (a mechanical oscillator), is shown in Fig.1. The mechanical oscillator, with a frequency of $\omega_{m}$ and an effective mass of $m_{e f f}$, is separately controlled by parametric driving on the spring constant $k(t)$ at a double frequency of $\omega_{m}$ and with a driving shift of $\delta$. Thus, the Hamiltonian of the driven parametric oscillator (DPO) is 26]

$$
\hat{H}_{o}=\frac{\hat{p}^{2}}{2 m_{e f f}}+\frac{1}{2}\left[k_{0}-k_{r} \sin \left(\omega_{d} t+2 \theta\right)\right] \hat{x}^{2},
$$

where the free spring constant is $k_{0}=m_{\text {eff }} \omega_{m}^{2}$, the driving frequency is $\omega_{d}=2\left(\omega_{m}-\delta\right)$, the driving amplitude is $k_{r}$, and $\theta$ is the driving-induced phase shift. For a classical DPO, strong squeezing of thermomechanical noise has been experimentally demonstrated [21]. To highlight the quantum squeezing effect, the original Hamiltonian $\hat{H}_{o}$ in a frame rotating at a frequency of $\omega_{m}-\delta$ can be written as [26]

$$
\hat{H}_{o r}=\hbar \delta \hat{b}^{\dagger} \hat{b}+i \frac{\hbar}{2}\left(\xi^{*} \hat{b}^{2}-\xi \hat{b}^{\dagger 2}\right)
$$

where $\hat{b}\left(\hat{b}^{\dagger}\right)$ is the phonon annihilation (creation) operator, with an effective mechanical frequency of $\delta$. The second term of $\hat{H}_{\text {or }}$ takes the exact form of a squeezing operator, which can generate quantum noise squeezing on the DPO. The squeeze parameter is $\xi=r e^{-2 i \theta}$, where the squeeze factor is $r=\omega_{m} k_{r} / 4 k_{0}$ and the phase shift $\theta$ adjusts the squeezing directions of the coupled quadratures [31. Therefore, the hybrid system depicted in Fig 1 can be described by the following Hamiltonian:

$$
\hat{H}_{h y b}=\hat{H}_{o r}+\hbar \delta_{c} \hat{a}^{\dagger} \hat{a}-\hbar g \hat{a}^{\dagger} \hat{a}\left(\hat{b}^{\dagger}+\hat{b}\right)+i \hbar\left(\eta \hat{a}^{\dagger}-\eta^{*} \hat{a}\right) .
$$

The second term of $\hat{H}_{h y b}$ represents a cavity mode $\hat{a}$ with a detuning of $\delta_{c}=\omega_{c a v}-\omega_{L}$, where $\omega_{c a v}$ and $\omega_{L}$ are the frequencies of the cavity mode and the pumping laser, respectively. The third term describes the optomechanical coupling between the cavity mode $\hat{a}$ and the mechanical mode $\hat{b}$, which has a coupling strength of $g$, and the last term represents the laser pumping with a strength of $\eta$ [32.

Because the entire system is subjected to fluctuations originating from both the external reservoirs and the internal quantum dynamics, the full motion of the system can be described by the quantum Langevin equations of $\hat{H}_{h y b}$, which contain noise terms for both the optical mode $\left(\hat{a}_{i n}\right)$ and the mechanical mode $\left(\hat{b}_{i n}\right)$ as follows:

$$
\begin{aligned}
& \frac{d \hat{a}}{d t}=-\left(i \delta_{c}+\frac{\kappa}{2}\right) \hat{a}+i g \hat{a}\left(\hat{b}^{\dagger}+\hat{b}\right)+\eta+\sqrt{\kappa} \hat{a}_{i n}, \\
& \frac{d \hat{b}}{d t}=-\left(i \delta+\frac{\Gamma_{m}}{2}\right) \hat{b}+i g \hat{a}^{\dagger} \hat{a}-\xi \hat{b}^{\dagger}+\sqrt{\Gamma_{m}} \hat{b}_{i n}
\end{aligned}
$$

where $\kappa$ is the total decay rate of the cavity mode and $\Gamma_{m}$ is the damping rate of the mechanical mode [32. The noise operators $\hat{a}_{i n}$ and $\hat{b}_{i n}$ represent the corresponding vacuum fluctuations, which have the following statistical reservoir properties: $\left\langle\hat{a}_{i n}(t) \hat{a}_{i n}^{\dagger}\left(t^{\prime}\right)\right\rangle=\left(n_{\text {cav }}^{\text {th }}+1\right) \delta\left(t-t^{\prime}\right)$, $\left\langle\hat{a}_{i n}^{\dagger}(t) \hat{a}_{i n}\left(t^{\prime}\right)\right\rangle=n_{\text {cav }}^{\text {th }} \delta\left(t-t^{\prime}\right),\left\langle\hat{b}_{i n}(t) \hat{b}_{i n}^{\dagger}\left(t^{\prime}\right)\right\rangle=\left(n_{m}^{\text {th }}+\right.$ 1) $\delta\left(t-t^{\prime}\right)$, and $\left\langle\hat{b}_{i n}^{\dagger}(t) \hat{b}_{i n}\left(t^{\prime}\right)\right\rangle=n_{m}^{t h} \delta\left(t-t^{\prime}\right)$. Here, we assume that the cavity field contains a number of thermal photons equal to $n_{\text {cav }}^{\text {th }}=\left[\exp \left(\hbar \omega_{\text {cav }} / k_{B} T\right)-1\right]^{-1}$ and that the number of thermal phonons is given by $n_{m}^{t h}=$ $\left[\exp \left(\hbar \omega_{m} / k_{B} T\right)-1\right]^{-1}[33$.

In the weak-pumping regime after cryogenic precooling, all dynamical quantities modulate around their classical equilibrium states as follows: $\hat{a}=a_{s}+\delta \hat{a}, \hat{a}^{\dagger}=$ $a_{s}^{*}+\delta \hat{a}^{\dagger}, \hat{b}=b_{s}+\delta \hat{b}$, and $\hat{b}^{\dagger}=b_{s}^{*}+\delta \hat{b}^{\dagger}$. Thus, the equilibrium position of the resonator can be determined using the following implicit equation:

$$
x_{s}=\frac{2 g \eta^{2}(\delta+r \sin 2 \theta)}{\left[(\kappa / 2)^{2}+\left(\delta_{c}-g x_{s}\right)^{2}\right]\left[\left(\Gamma_{m} / 2\right)^{2}+\delta^{2}-r^{2}\right]},
$$

where $x_{s}=b_{s}+b_{s}^{*}$. In Fig, 2 , a squeezing-enhanced sensitive displacement of $x_{s}$ near the critical squeezing point of $r \sim r_{c}=\sqrt{\delta^{2}+\Gamma_{m}^{2} / 4}$ is identified, and it implies an efficient method of phonon cooling when $r>r_{c}$ [32. The 


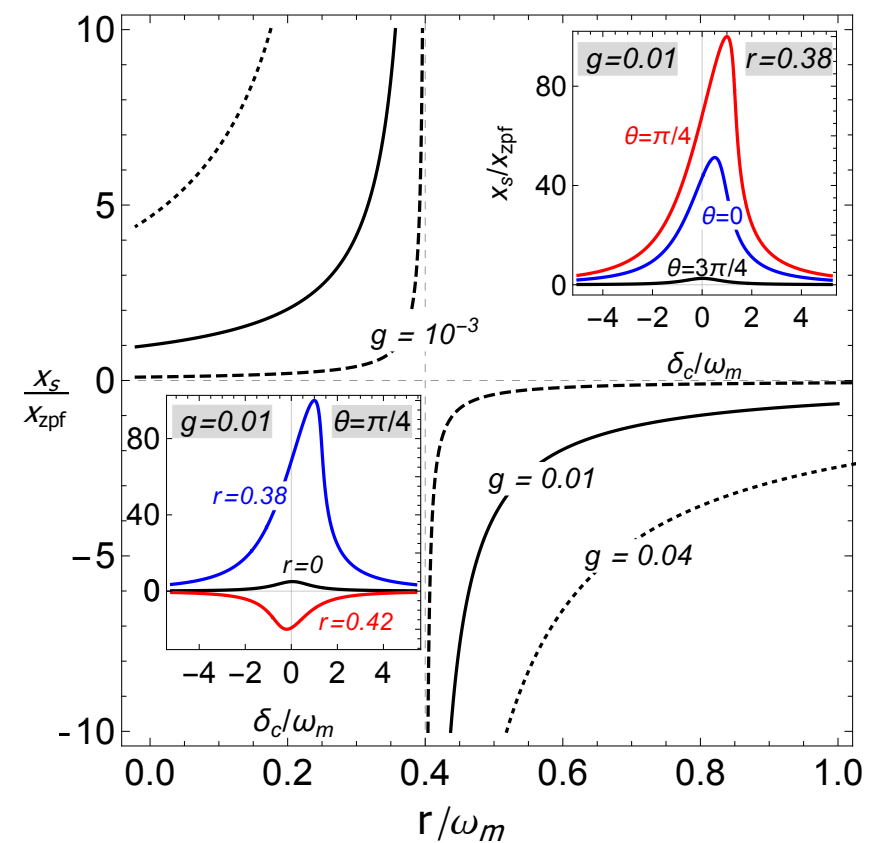

FIG. 2: The static displacements of the DPO modified by the squeeze factor $r(\theta=\pi / 4)$ with different optomechanical coupling rates $g$. Insets: The displacement responses of $x_{s}$ with respect to the pumping detuning $\delta_{c}$ under different squeezing parameters. The other parameters are $\delta_{c}=2, \kappa=2, \delta=0.4$, $\Gamma_{m}=10^{-3}$, and $\eta=10$, all scaled by $\omega_{m}$.

nonlinear static responses of $x_{s}$ with respect to the pumping detuning $\delta_{c}$ also sensitively depend on $r$ (lower inset) and $\theta$ (upper inset). To ensure reliable performance, the stability requirement of the DPO imposes an upper bound on the squeezing degree of $r$ such that $r<r_{c}$ for steady-state parametric squeezing [28, 32. However, the instability beyond the squeezing bound can be overcome in two ways. One is to use a red-detuned cooling laser to induce a positive damping rate $\Gamma_{\text {opt }}$ on the DPO (through optical spring and damping effects), which can stabilize the steady-state squeezing over a large squeezing region [34. The other reliable technique is to introduce quantum feedback control [35, 36] over the dynamics of the DPO that extends beyond its steady-state performance (e.g., locking onto a controlled self-sustained oscillation) and can work in the deep squeezing regime [26].

For simplicity, we introduce the replacements $\delta \hat{a} \rightarrow \hat{a}$ and $\delta \hat{b} \rightarrow \hat{b}$ to obtain an effective linearized Hamiltonian of the system:

$$
\begin{aligned}
\hat{H}_{e f f}= & \hbar \Delta_{c} \hat{a}^{\dagger} \hat{a}+\hbar \delta \hat{b}^{\dagger} \hat{b}-\hbar g\left(a_{s}^{*} \hat{a}+a_{s} \hat{a}^{\dagger}\right)\left(\hat{b}^{\dagger}+\hat{b}\right) \\
& +i \frac{\hbar}{2}\left(\xi^{*} \hat{b}^{2}-\xi \hat{b}^{\dagger 2}\right)
\end{aligned}
$$

where $\Delta_{c}$ is the position-shifted cavity detuning and is defined as $\Delta_{c} \equiv \delta_{c}-g x_{s}$. Clearly, the last term of Eq.(7) generates noise squeezing on the mechanical mode $\hat{b}$ by means of the squeeze operator $\hat{S}(\xi)=\exp \left(\frac{1}{2} \xi^{*} \hat{b}^{2}-\frac{1}{2} \xi \hat{b}^{\dagger 2}\right)$, where $\xi=r e^{-2 i \theta}$ controls the degree $r$ and the angle $\theta$ of the squeezing on the coupled quadratures [31.

With the physical setup described above, our system can simultaneously apply mechanical squeezing to one quadrature and perform cooling on the other. The squeezing-and-cooling effect can be analyzed based on the phonon noise spectrum of the DPO. The final number of phonons in the mechanical resonator is determined by

$$
\bar{n}=\left\langle\hat{b}^{\dagger}(t) \hat{b}(t)\right\rangle=\frac{1}{2 \pi} \int_{-\infty}^{\infty} S_{n}(\omega) d \omega,
$$

where $S_{n}(\omega)$ is the phonon-number spectrum [32, and this implies an effective cooling temperature $T_{\text {eff }}$ of

$$
T_{e f f}(\bar{n})=\frac{\hbar \omega_{m}}{\ln \left(\frac{1}{\bar{n}}+1\right)} .
$$

The above relation is derived from the detailed balance expression [8, 12, 33], and $\bar{n}$ is equal to the area underneath the spectral curve $S_{n}(\omega)$, implying that $\lim _{\bar{n} \rightarrow 0} T_{\text {eff }} \rightarrow 0$.

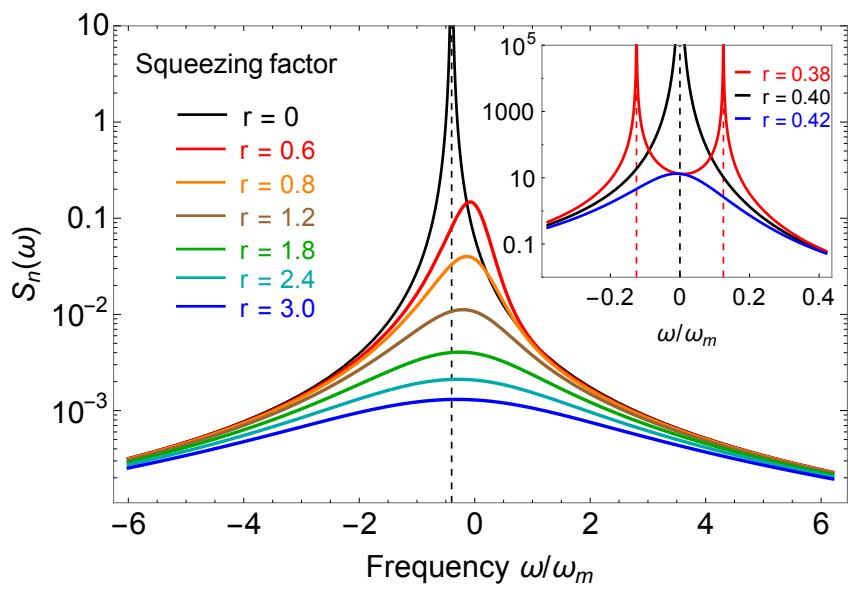

FIG. 3: The phonon-number spectra of the mechanical resonator for different squeeze factors $r>r_{c}$ (scaled by $\omega_{m}$ ) with a fixed squeezing angle of $\theta=\pi / 4$. Inset: phonon spectra for squeeze factors $r$ near the critical squeezing factor $r_{c} \approx 0.4$. The effective mechanical frequency is $\delta=0.4$ (indicated by the dashed vertical line), the optomechanical coupling rate is $g=0.001$, the average thermal occupation is $n_{m}^{t h}=10$, and the optical-mode occupation is $n_{\text {cav }}^{\text {th }}=1$. The other parameters are $\delta_{c}=2, \kappa=2, \Gamma_{m}=10^{-3}$, and $\eta=10$, all scaled by $\omega_{m}$.

In Fig 3 , we present the calculated phonon-number spectra $S_{n}(\omega)$ for different squeeze factors $r$. The spectra exhibit a significant decrease in $T_{\text {eff }}$ with an increasing squeeze factor $r$. The inset figure reveals the critical squeeze factor $r_{c}$ and the marked squeezing-based cooling that occurs when $r>r_{c}$ 32. Here, we have chosen moderate parameters that can be easily realized in optomechanical systems [17. A high- $Q$ resonator with $\Gamma_{m} / \omega_{m}=0.001$ is used to support the squeezing performance, and a precooling process is applied that achieves 
an average thermal occupation of $n_{m}^{t h}=10$ to enhance the noise squeezing effect. The cavity mode, with an occupation of $n_{c a v}^{t h}=1$, should be pumped by a far-red-detuned low-power laser source. These undemanding conditions for squeezing-based cooling can be easily fulfilled in a bad cavity $\left(\kappa / \omega_{m}=2\right)$ beyond the resolved-sideband cooling limit and in the weak-coupling regime $(g<\kappa)$. The cooling mechanism takes effect in this regime because the squeezed "hotter" phonons with enhanced fluctuations are squeezed out and quickly taken away by the coupled photons leaking from the bad cavity. Because a larger squeeze factor $r$ (the lower spectra shown in Fig 3) will drive the system into an unstable state, this technique is limited only by the squeeze factor $r / \omega_{m}=k_{r} / 4 k_{0}$ that is determined by the relative modulation amplitude of the parametric resonator. Regardless, a small squeeze factor of $r_{c}<r<2$ is still effective because we can use successive squeezing-and-cooling cycles to achieve a lower temperature 36.

Another interesting property of squeezing-based cooling is that the cooling will depend on the squeezing angle $\theta$ when the system enters the strong-coupling regime of $g \sim \kappa$ under a higher degree of squeezing. This dependence arises because the light mode couples to the asymmetric quadratures, whose squeezing directions are modified by the angle $\theta$. From the perspective of the squeezing picture, the Hamiltonian given in (7) will be $\hat{S}^{\dagger}(\xi) \hat{H}_{\text {eff }} \hat{S}(\xi)$, and the third term of Eq. (7), which describes the optical coupling of the mode $\hat{a}$ to the mechanical quadrature of $\hat{X}$ defined by $\hat{b}=(\hat{X}+i \hat{P}) / 2$, becomes $(\cosh r-\cos 2 \theta \sinh r) \hat{X}+(\sin 2 \theta \sinh r) \hat{P}$. This expression clearly shows a $\theta$ dependence of the coupling of the quadrature to the cavity mode. Fig 4 shows how the phonon spectrum changes with respect to the squeezing angle $\theta$ when the squeeze factor $r$ is fixed. The black curve is the phonon spectrum without squeezing $(r=0)$, and the other curves represent the phonon spectra under a fixed squeeze factor of $r / \omega_{m}=1.2$ and different squeezing angles. The results verify the $\theta$ dependence of the cooling rate, and an optimal case arises when the cooling laser is coupled directly to the "hottest" quadrature with the maximum fluctuation. Both mechanical resonant peaks at $\pm r_{c} / \omega_{m}$ appearing in Fig 4 (indicated by vertical dashed lines) are due to an increase in the coupling $g$. The weak phonon resonant peak at $+r_{c} / \omega_{m}$ will be suppressed by the squeezing effect even in the strong-coupling regime. Moreover, a phonon spectrum with a richer structure within the resolved-sideband limit or in the strong-coupling regime still exhibits a similar squeeze-induced enhancement of the cooling effect 32 .

In summary, through direct numerical calculations based on a simple physical picture, we have revealed a deep cooling scheme for an optomechanical resonator in which the cooling performance is dramatically enhanced by the squeezing effect induced by parametric amplification. We have demonstrated that by increasing the squeeze factor $r$, we can effectively reduce the area under the phonon-number spectral curve, thereby extracting

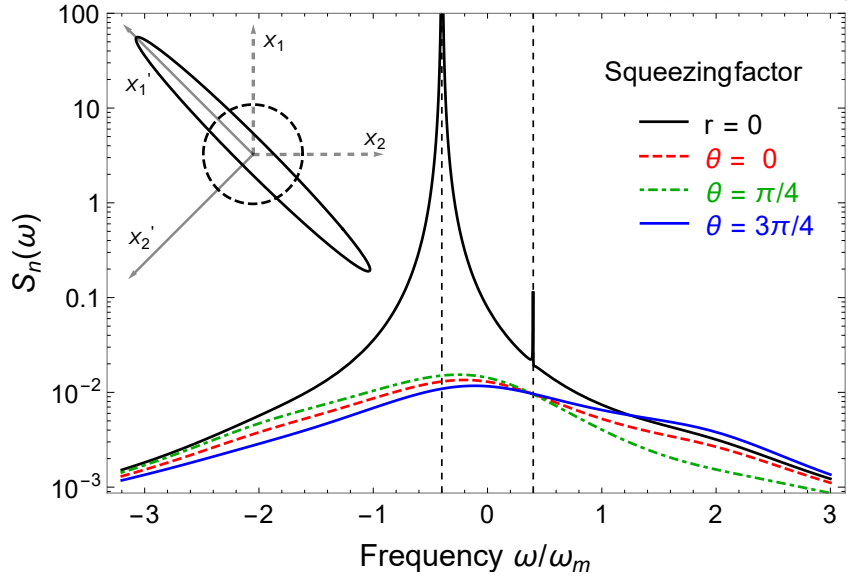

FIG. 4: The phonon-number spectral curves of the mechanical resonator without squeezing (black line for $r=0$ ) and under squeezing with a fixed squeeze factor of $r=1.2$ for different squeezing angles $\theta$. Inset: the noise error ellipses of the two quadratures for $r=0$ (dashed circle) and for $r=1.2$ (solid ellipse). The optomechanical coupling rate $g$ is improved to 0.01 , and the other parameters are the same as in Fig 3

significant "heat" from the squeezed motion of a mechanical oscillator, to reach an effective temperature below the quantum shot noise. The idea proposed here is that one quadrature of the mechanical mode can be "heated" by squeezing the other to improve the cooling capacity by coupling the cooling laser directly to the "heated" quadrature, allowing the "hotter" phonons to be quickly taken away by the leakage of photons from a bad cavity. This method can be used to rapidly cool the mechanical motion down to its quantum ground state, beyond the standard quantum limit. The resulting high cooling efficiency is of interest for many quantum applications, such as quantum precision measurement or quantum sensors and rapid state initialization for quantum processing. We believe that this squeezing-based cooling scheme can serve as a universal technique for facilitating the development of quantum technology for use in macroscopic solid-state systems.

\section{Acknowledgments}

We thank Keye Zhang and the other active fellows in our laboratory for valuable discussions. This work was supported by the National Natural Science Foundation of China (Grant No. 11447025) and the Scientific Research Foundation for Returned Overseas Chinese Scholars of the State Education Ministry. 
[1] M. Aspelmeyer, T. J. Kippenberg, and F. Marquardt, Rev. Mod. Phys. 86, 1391 (2014).

[2] E. F. Nichols and G. F. Hull, Astrophys. J. 17, 315 (1903).

[3] D. Kleckner, W. Marshall, M. J. A. de Dood, K. N. Dinyari, B-J Pors, W. T. M. Irvine, and D. Bouwmeester, Phys. Rev. Lett. 96, 173901 (2006).

[4] H. Rokhsari, T. J. Kippenberg, T. Carmon, and K. J. Vahala, Opt. Express 13, 5293 (2005).

[5] Michael Metcalfe, Appl. Phys. Rev. 1, 031105 (2014).

[6] A. Schliesser, P. Del'Haye, N. Nooshi, K. J. Vahala, and T. J. Kippenberg, Phys. Rev. Lett. 97, 243905 (2006).

[7] S. Gigan, H. R. Bohm, M. Paternostro, F. Blaser, G. Langer, J. B. Hertzberg, K. C. Schwab, D. Buerle, M. Aspelmeyer, and A. Zeilinger, Nature (London) 444, 67 (2006).

[8] F. Marquardt, J. P. Chen, A. A. Clerk, and S.M. Girvin, Phys. Rev. Lett. 99, 093902 (2007).

[9] R. W. Peterson, T. P. Purdy, N. S. Kampel, R. W. Andrews, P.-L. Yu, K. W. Lehnert, and C. A. Regal, Phys. Rev. Lett. 116, 063601 (2016).

[10] V. B. Braginsky and S. P. Vyatchanin, Phys. Lett. A 293, 228 (2002).

[11] O. Arcizet, P. F. Cohadon, T. Briant, M. Pinard, and A. Heidmann1, Nature (London) 444, 71 (2006).

[12] A. A. Clerk, M. H. Devoret, S. M. Girvin, Florian Marquardt, and R. J. Schoelkopf, Rev. Mod. Phys. 82, 1155 (2010).

[13] A. Schliesser, O. Arcizet, R. Rivière, G. Anetsberger, and T. J. Kippenberg, Nat. Phys 5, 509 (2009).

[14] Y.-S. Park and H. Wang, Nat. Phys. 5, 489 (2009).

[15] J. D. Teufel, T. Donner, D. Li, J. H. Harlow, M. S. Allman, K. Cicak, A. J. Sirois, J. D. Whittaker, K. W. Lehnert, and R. W. Simmonds, Nature (London) 475, 359 (2011).

[16] J. Chan, T. P. Mayer Alegre, A. H. Safavi-Naeini, J. T. Hill, A. Krause, S. Groeblacher, M. Aspelmeyer, and O. Painter, Nature (London) 478, 89 (2011).

[17] J. B. Clark, F. Lecocq, R. W. Simmonds, J. Aumentado, and J. D. Teufel, Nature (London) 541, 191 (2017).

[18] The relation between the fluctuation (energy variance $\Delta E$ ) and temperature $(T)$ of a classical system in equilibrium with a bath can be expressed, according to the fluctuation-dissipation theorem, as $\Delta E=\mu k_{B} T$, where $\mu$ is the mobility for the energy damping to the equilibrium bath (e.g., the energy fluctuation in a canonical ensemble satisfies $\left.\Delta E \propto k_{B} T\right)$. However, for a well-isolated quantum system, the relation is ambiguous because of the variety of temperature definitions beyond that with respect to a bath. Nevertheless, the fluctuation-dissipation theorem is assumed to be valid in the quantum regime [37, and thus, we use the word "heated" in the sense of an effective temperature $T_{\text {eff }}$ related to the energy fluctuations of the system.

[19] C. M. Caves, Phys. Rev. D 23, 1693 (1981).

[20] Yamamoto and Haus, Rev. Mod. Phys. 58, 1001 (1986).

[21] D. Rugar and P. Grutter, Phys. Rev. Lett. 67, 699 (1991).

[22] M. A. Castellanos-Beltran, K. D. Irwin, G. C. Hilton, L. R. Vale, and K. W. Lehnert, Nat. Phys 4, 929 (2008).

[23] M. J. Woolley, A. C. Doherty, G. J. Milburn, and K. C. Schwab, Phys. Rev. A 78, 062303 (2008).

[24] E. E. Wollman, C. U. Lei, A. J. Weinstein, J. Suh, A. Kronwald, F. Marquardt, A. A. Clerk, K. C. Schwab, Science 349, 952 (2015).

[25] J.-M. Pirkkalainen, E. Damskägg, M. Brandt, F. Massel, and M. A. Sillanpää, Phys. Rev. Lett. 115, 243601 (2015).

[26] A. Szorkovszky, A. C. Doherty, G. I. Harris, and W. P. Bowen, Phys. Rev. Lett. 107, 213603 (2011).

[27] J-Q. Liao and C. K. Law, Phys. Rev. A 83, 033820 (2011).

[28] A. Szorkovszky, G. A. Brawley, A. C. Doherty, and W. P. Bowen, Phys. Rev. Lett. 110, 184301 (2013).

[29] G. S. Agarwal and S. Huang, Phys. Rev. A 93, 043844 (2016).

[30] S. Huang and G. S. Agarwal, Phys. Rev. A 79, 013821 (2009).

[31] D. F. Walls and G. J. Milburn, Quantum Optics (Springer, New York, 1995).

[32] See the Supplemental Materials for the details of the calculations of the theoretical model, the stability analysis, the cirtical squeeze factor and the phonon spectrum in the strong-coupling regime.

[33] W. P. Bowen and G. J. Milburn, Quantum Optomechanics (CRC Press, New York, 2016).

[34] S. W. Schediwy, C. Zhao, L. Ju, D. G. Blair, and P. Willems, Phys. Rev. A 77, 013813 (2008).

[35] D. J. Wilson, V. Sudhir, N. Piro, R. Schilling, A. Ghadimi and T. J. Kippenberg, Nature 524, 325 (2015).

[36] M. Rossi, N. Kralj, S. Zippilli, R. Natali, A. Borrielli, G. Pandraud, E. Serra, G. DiGiuseppe, D. Vitali, Phys. Rev. Lett. 119, 123603 (2017).

[37] M. Gring, M. Kuhnert, T. Langen, T. Kitagawa, B. Rauer, M. Schreitl, I. Mazets, D. Adu Smith, E. Demler, J. Schmiedmayer, Science 337, 1318 (2012). 